\title{
Externe Dienstleister: Neues zur Frage einer Sozialversicherungspflicht
}

\author{
Ist eine selbstständig in Zahnarztpraxen tätige zahnmedizinische Verwaltungsassistentin nicht \\ doch als sozialversicherungspflichtige Arbeitnehmerin einzustufen? Mit dieser Frage hat sich das \\ Landessozialgericht (LSG) Baden-Württemberg befasst (Beschluss vom 08.07.2016, Az. L 4 R \\ 4979/15). Das Thema beschäftigt die Gerichte zunehmend, sodass es umso wichtiger ist, sich mit \\ dieser Thematik auseinanderzusetzen, wenn externe Dienstleister in der Praxis eingesetzt werden.
}

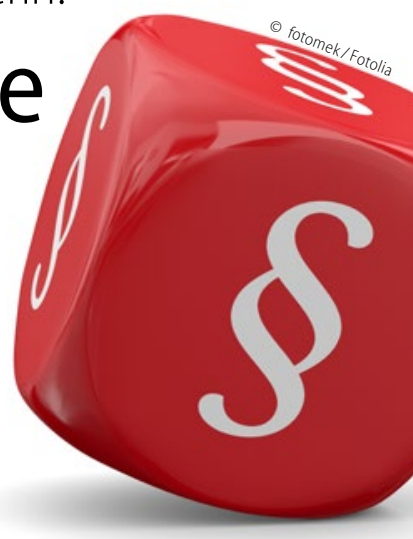

\section{Der Fall}

In dem konkreten Fall setzte eine niedergelassene Zahnärztin eine geprüfte zahnmedizinische Verwaltungsassistentin in ihrer Praxis ein, die an ihrem Wohnsitz ein Gewerbe im Bereich "Zahnmedizinische Verwaltungstätigkeit" mit Büro angemeldet hat. Die Verwaltungsassistentin erbrachte dabei Praxisverwaltungstätigkeiten für sechs Zahnarztpraxen. Für die Zahnärztin war sie wegen eines mündlich abgeschlossenen Vertrages tätig, wobei sie für ihre Tätigkeit jeweils monatlich aufgeschlüsselt eine Rechnung nach geleisteten Zeitstunden erstellte.

\section{Die Entscheidung}

In dem konkreten Fall wurde die Tätigkeit von dem zuständigen Sozialversicherungsträger als sozialversicherungspflichtig eingestuft, woraufhin die betroffene Zahnärztin erfolgreich Klage vor dem Sozialgericht Reutlingen erhob. Auch nach Auffassung des LSG Baden-Württemberg übte sie ihre Tätigkeit bei der Zahnärztin selbstständig aus, womit sie in dieser Tätigkeit nicht versicherungspflichtig in der gesetzlichen Rentenversicherung und nach dem Recht der Arbeitsförderung gewesen sei.

Versicherungspflichtig seien in der Rentenversicherung und in der Arbeitslosenversicherung gegen Arbeitsentgelt beschäftigte Personen. Beschäftigung sei nach $₫ 7$ Abs. 1 Satz 1 SGB IV die nichtselbstständige Arbeit, insbesondere in einem Arbeitsverhältnis.

\section{Weisungsrecht erforderlich}

Nach der ständigen Rechtsprechung des BSG setze eine Beschäftigung voraus, dass der Arbeitnehmer vom Arbeitgeber persönlich abhängig sei. Bei einer Beschäftigung in einem fremden Betrieb sei dies der Fall, wenn der Beschäftigte in den Betrieb eingegliedert sei und dabei einem Zeit, Dauer, Ort und Art der Ausführung umfassenden Weisungsrecht des Arbeitgebers unterliege.

Maßgebend sei das Gesamtbild der Arbeitsleistung, das sich nach den tatsächlichen Verhältnissen bestimme. Vorliegend sei die Verwaltungshelferin wegen des mündlich geschlossenen, für beide Seiten jederzeit kündbaren Vertrages verpflichtet gewesen, für die Zahnärztin Praxismanagementtätigkeiten zu erbringen, insbesondere Abrechnungen durchzuführen sowie Kostenpläne und Rechnungen zu erstellen.

Ein (arbeitsrechtliches) Weisungsrecht habe nicht bestanden. Dies gelte zum einen in zeitlicher und örtlicher Hinsicht. Eine Eingliederung in die Arbeitsorganisation der Zahnärztin habe auch nicht in personeller Hinsicht bestanden. Die Tätigkeit sei ohne Zusammenarbeit mit den Beschäftigten der Zahnärztin verrichtet worden. In fachlicher Hinsicht habe ebenfalls kein Weisungsrecht bestanden. Auch die Vergütungsregelung spreche für eine selbstständige Tätigkeit [Stundenhonorar (nur) für jede tatsächlich geleistete Arbeitsstunde]. Die Vergütung nur tatsächlich geleisteter Stunden spreche gegen das Vorliegen einer abhängigen Beschäftigung (BSG, Urteil vom 12. Februar 2004, B 12 KR 26/02 R). (\$\$167 ff. SGB III).

\section{Mehrere Auftraggeber}

Für eine selbstständige Tätigkeit spreche auch, dass die Verwaltungshelferin weitere Auftraggeber, nämlich fünf weitere Zahnarztpraxen hatte, für die sie gleichgelagerte Tätigkeiten erbrachte, sowie weitere Praxen, in denen sie Schulungen durchführte. Zwar sei für jedes Vertragsverhältnis die sozialversicherungsrechtliche Beurteilung gesondert vorzunehmen, jedoch spreche der Umstand, für mehrere Auftraggeber tätig zu sein, für eine selbstständige Tätigkeit, nicht zuletzt, weil sie die wirtschaftliche Abhängigkeit von einem Auftraggeber oder Arbeitgeber reduziere oder gar aufhebe.

\section{Bewertung}

Die Entscheidung setzt einen deutlichen Kontrapunkt zur Entscheidung des LSG Berlin-Brandenburg vom 29.01.2016 (L 1 KR 118/14), das die Tätigkeit einer selbstständigen Abrechnungsspezialistin als sozialversicherungspflichtig eingestuft hatte. Eine höchstrichterliche Klärung steht aus, was für mehr Rechtsklarheit sorgen würde. Im Fall vor dem LSG Baden-Württemberg wurde die Revision nicht zugelassen. Bei der Einbindung externer Abrechnungsmitarbeiter empfiehlt es sich abzuklären, ob die vertraglichen Regelungen den vom LSG Baden-Württemberg aufgestellten Kriterien gerecht werden. Unabhängig davon ist es bei dem Einsatz externer Dienstleister sehr wichtig, dass die Schweigepflicht gegenüber dem Patienten gewahrt wird und ein Einverständnis des Patienten hinsichtlich seiner höchstpersönlichen Daten eingeholt wird, wenn hierin eine Einsichtnahme erfolgt.

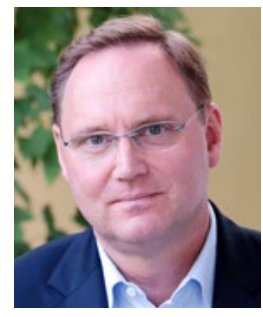

RA Michael Lennartz

www.lennmed.de 